\title{
Generalizing the Ramsey Problem through Diameter
}

\author{
Dhruv Mubayi* \\ Submitted: January 8, 2001; Accepted: November 13, 2001. \\ MR Subject Classifications: 05C12, 05C15, 05C35, 05C55
}

\begin{abstract}
Given a graph $G$ and positive integers $d, k$, let $f_{d}^{k}(G)$ be the maximum $t$ such that every $k$-coloring of $E(G)$ yields a monochromatic subgraph with diameter at most $d$ on at least $t$ vertices. Determining $f_{1}^{k}\left(K_{n}\right)$ is equivalent to determining classical Ramsey numbers for multicolorings. Our results include

- determining $f_{d}^{k}\left(K_{a, b}\right)$ within 1 for all $d, k, a, b$

- for $d \geq 4, f_{d}^{3}\left(K_{n}\right)=\lceil n / 2\rceil+1$ or $\lceil n / 2\rceil$ depending on whether $n \equiv 2(\bmod 4)$ or not

- $f_{3}^{k}\left(K_{n}\right)>\frac{n}{k-1+1 / k}$

The third result is almost sharp, since a construction due to Calkin implies that $f_{3}^{k}\left(K_{n}\right) \leq \frac{n}{k-1}+k-1$ when $k-1$ is a prime power. The asymptotics for $f_{d}^{k}\left(K_{n}\right)$ remain open when $d=k=3$ and when $d \geq 3, k \geq 4$ are fixed.
\end{abstract}

\section{Introduction}

The Ramsey problem for multicolorings asks for the minimum $n$ such that every $k$-coloring of the edges of $K_{n}$ yields a monochromatic $K_{p}$. This problem has been generalized in many ways (see, e.g., $[2,6,7,9,12,13,14])$. We begin with the following generalization due to Paul Erdős [8] (see also [11]):

Problem 1 What is the maximum $t$ with the property that every $k$-coloring of $E\left(K_{n}\right)$ yields a monochromatic subgraph of diameter at most two on at least $t$ vertices?

A related problem is investigated in [14], where the existence of the Ramsey number is proven when the host graph is not necessarily a clique. Call a subgraph of diameter at most $d$ a $d$-subgraph.

*Department of Mathematics, Statistics, and Computer Science, University of Illinois at Chicago, 851 S. Morgan Street, Chicago, IL 60607-7045, mubayi@math.uic.edu

Keywords: diameter, generalized ramsey theory 
Theorem 2 (Tonoyan [14]) Let $D, k \geq 1, d \geq D, n \geq 2$. Then there is a smallest integer $t=R_{D, k}(n, d)$ such that every graph $G$ with diameter $D$ on at least $t$ vertices has the following property: every $k$-coloring of $E(G)$ yields a monochromatic d-subgraph on at least $n$ vertices.

We study a problem closely related to Tonoyan's result that also generalizes Problem 1 to larger diameter.

Definition 3 Let $G$ be a graph and $d, k$ be positive integers. Then $f_{d}^{k}(G)$ is the maximum $t$ with the property that every $k$-coloring of $E(G)$ yields a monochromatic d-subgraph on at least $t$ vertices.

The asymptotics for $f_{d}^{k}(G)$ when $G=K_{n}$ and $d=2$ (Erdős' problem) were determined in [10].

Theorem 4 (Fowler [10]) $f_{2}^{2}\left(K_{n}\right)=\lceil 3 n / 4\rceil$ and if $k \geq 3$, then $f_{2}^{k}\left(K_{n}\right) \sim n / k$ as $n \rightarrow \infty$.

In this paper, we study $f_{d}^{k}(G)$ when $G$ is a complete graph or a complete bipartite graph. In the latter case, we determine its value within 1.

Theorem 5 (Section 3) Let $k, a, b \geq 2$. Then $f_{2}^{k}\left(K_{a, b}\right)=1+\lceil\max \{a, b\} / k\rceil$, and for $d \geq 3$,

$$
\left\lceil\frac{1}{a b}\left(\left\lceil\frac{a b^{2}}{k}\right\rceil+\left\lceil\frac{a^{2} b}{k}\right\rceil\right)\right\rceil \leq f_{d}^{k}\left(K_{a, b}\right) \leq\left\lceil\frac{a}{k}\right\rceil+\left\lceil\frac{b}{k}\right\rceil .
$$

Determining $f_{d}^{k}\left(K_{n}\right)$ (for $d \geq 3$ ) seems more difficult. We succeed in doing this only when $d>k=3$.

Theorem 6 (Section 4) Let $d \geq 4$. Then

$$
f_{d}^{3}\left(K_{n}\right)= \begin{cases}n / 2+1 & n \equiv 2(\bmod 4) \\ \lceil n / 2\rceil & \text { otherwise }\end{cases}
$$

When $d=3$ we are able to obtain bounds for $f_{d}^{k}\left(K_{n}\right)$.

Theorem 7 (Section 5) Let $k \geq 2$. Then $f_{3}^{k}\left(K_{n}\right)>n /(k-1+1 / k)$.

In section 5 we also describe an unpublished construction of Calkin which implies that $f_{3}^{k}\left(K_{n}\right) \leq n /(k-1)+k-1$ when $k-1$ is a prime power. This shows that the bound in Theorem 7 is not far off from being best possible. In section 6 we summarize the known results for $f_{d}^{k}\left(K_{n}\right)$. Our main tool for Theorems 5 and 7 is developed in Section 2. 


\section{The Main Lemma}

In this section we prove a statement about 3-subgraphs in colorings of bipartite graphs. Although this is later used in the proofs of Theorems 5 and 7 , we feel it is of independent interest.

Suppose that $G$ is a graph and $c: E(G) \rightarrow[k]$ is a $k$-coloring of its edges. For each $i \in[k]$ and $x \in V(G)$, let $N_{i}(x)=\{y \in N(x): c(x y)=i\}$ and $d_{i}(x)=\left|N_{i}(x)\right|$. For $u v \in E(G)$, let the weight of $u v$ be

$$
w(u v)=d_{c(u v)}(u)+d_{c(u v)}(v) .
$$

Lemma 8 Let $G$ be a subgraph of $K_{a, b}$ with e edges and $d \geq 3$. Then

$$
f_{d}^{k}(G) \geq\left\lceil\frac{1}{e}\left(\left\lceil\frac{e^{2}}{a k}\right\rceil+\left\lceil\frac{e^{2}}{b k}\right\rceil\right)\right\rceil \geq\left\lceil\frac{e}{a k}\right\rceil+\left\lceil\frac{e}{b k}\right\rceil-1
$$

Proof: Suppose that $K_{a, b}$ has bipartition $A, B$ with $X=V(G) \cap A$, and $Y=V(G) \cap B$. Let $c: E(G) \rightarrow[k]$ be a $k$-coloring. Observe that an edge with weight $w$ gives rise to a 3-subgraph on $w$ vertices. We prove the stronger statement that $G$ has an edge with weight at least

$$
\left\lceil\frac{1}{e}\left(\left\lceil\frac{e^{2}}{a k}\right\rceil+\left\lceil\frac{e^{2}}{b k}\right\rceil\right)\right\rceil
$$

We obtain a lower bound on the sum of all the edge-weights.

$$
\begin{aligned}
\sum_{u v \in E(G)} w(u v) & =\sum_{x \in X} \sum_{i \in[k]} \sum_{y \in N_{i}(x)} w(x y) \\
& =\sum_{x \in X} \sum_{i \in[k]} \sum_{y \in N_{i}(x)} d_{i}(x)+d_{i}(y) \\
& =\sum_{x \in X} \sum_{i \in[k]}\left[d_{i}(x)\right]^{2}+\sum_{y \in Y} \sum_{i \in[k]}\left[d_{i}(y)\right]^{2} \\
& \geq\left\lceil\frac{e^{2}}{a k}\right\rceil+\left\lceil\frac{e^{2}}{b k}\right],
\end{aligned}
$$

where (1) follows from the Cauchy-Schwarz inequality applied to each double sum. Since there is an edge with weight at least as large as the average, we have

$$
f_{d}^{k}(G) \geq\left\lceil\frac{1}{e}\left(\left\lceil\frac{e^{2}}{a k}\right\rceil+\left\lceil\frac{e^{2}}{b k}\right\rceil\right)\right\rceil \geq\left\lceil\frac{e}{a k}+\frac{e}{b k}\right\rceil \geq\left\lceil\frac{e}{a k}\right\rceil+\left\lceil\frac{e}{b k}\right\rceil-1 .
$$

A slight variation of the proof of Lemma 8 also yields the following more general result.

Lemma 9 Suppose that $G$ is a graph with $n$ vertices and e edges. Let $c: E(G) \rightarrow[k]$ be a $k$-coloring of $E(G)$ such that every color class is triangle-free. Then $G$ contains a monochromatic 3-subgraph on at least $4 e /(n k)$ vertices. 


\section{Bipartite Graphs}

Proof of Theorem 5: Let $c: E\left(K_{a, b}\right) \rightarrow[k]$ be a $k$-coloring. The lower bound for the case $d=2$ is obtained by considering a pair $(v, i)$ for which $d_{i}(v)$ is maximized. The set $v \cup N_{i}(v)$ induces a monochromatic 2-subgraph. The lower bound when $d \geq 3$ follows from Lemma 8. For the upper bounds we provide the following constructions.

Let $K_{a, b}$ have bipartition $X=\left\{x_{1}, \ldots, x_{a}\right\}$ and $Y=\left\{y_{1}, \ldots, y_{b}\right\}$, and assume that $a \leq b$. Partition $X$ into $k$ sets $X_{1}, \ldots, X_{k}$, each of size $\lceil a / k\rceil$ or $\lfloor a / k\rfloor$, and partition $Y$ into $k$ sets $Y_{1}, \ldots, Y_{k}$, each of size $\lceil b / k\rceil$ or $\lfloor b / k\rfloor$. Furthermore, let both these partitions be "consecutive" in the sense that $X_{1}=\left\{x_{1}, x_{2}, \ldots, x_{r}\right\}, X_{2}=\left\{x_{r+1}, x_{r+2} \ldots, x_{r+s}\right\}$, etc. Finally, for each nonnegative integer $t$, let $Y_{i}+t=\left\{y_{l+t}: y_{l} \in Y_{i}\right\}$, where subscripts are taken modulo $b$.

When $d=2$ and $j \in[k]$, let the $j^{\text {th }}$ color class be all edges between $x_{i}$ and $Y_{j}+(i-1)$ for each $i \in[n]$. Because $K_{a, b}$ is bipartite, the distance between a pair of nonadjacent vertices $x \in X$ and $y \in Y$ in the subgraph formed by the edges in color $j$ is at least three. Thus a 2-subgraph of $K_{a, b}$ is a complete bipartite graph.

For $1 \leq i \leq k$, let $\alpha_{i}$ be the smallest subscript of an element in $Y_{i}$. Thus $\alpha_{i+1}-\alpha_{i}=\left|Y_{i}\right|$ since $Y_{i}=\left\{y_{\alpha_{i}}, y_{\alpha_{i}+1}, \ldots, y_{\alpha_{i+1}-1}\right\}$. Fix $l \in[k]$ and let $H$ be a largest monochromatic complete bipartite graph in color $l$. Let $A=V(H) \cap X$ and $B=V(H) \cap Y$. Let $r$ be the smallest index such that $x_{r} \in A$ and let $s$ be the largest index such that $x_{s} \in A$. As $N_{H}\left(x_{r}\right)=\left\{y_{\alpha_{l}+r-1}, y_{\alpha_{l}+r}, \ldots, y_{\alpha_{l+1}+r-2}\right\}$ and $N_{H}\left(x_{s}\right)=\left\{y_{\alpha_{l}+s-1}, y_{\alpha_{l}+s}, \ldots, y_{\alpha_{l+1}+s-2}\right\}$, we have $N_{H}\left(x_{r}\right) \cap N_{H}\left(x_{s}\right)=\left\{y_{\alpha_{l}+s-1}, \ldots, y_{\alpha_{l+1}+r-2}\right\}$, where subscripts are taken modulo $b$. Consequently,

$$
\begin{aligned}
|V(H)| & \leq\left|\left\{x_{r}, \ldots, x_{s}\right\}\right|+\left|\left\{y_{\alpha_{l}+s-1}, \ldots, y_{\alpha_{l+1}+r-2}\right\}\right| \\
& =(s-r+1)+\left(\alpha_{l+1}+r-2-\left(\alpha_{l}+s-1\right)+1\right) \\
& =1+\alpha_{l+1}-\alpha_{l} \\
& =1+\left|Y_{l}\right| \\
& \leq 1+\lceil b / k\rceil .
\end{aligned}
$$

When $d>2$ and $j \in[k]$, let the $j^{t h}$ color class consist of the edges between $X_{i}$ and $Y_{i-1+j}$ (subscripts taken modulo $k$ ) for each $i \in[k]$. The maximum size of a connected monochromatic subgraph is $\max _{i, i^{\prime}}\left\{\left|X_{i}\right|+\left|Y_{i^{\prime}}\right|\right\}=\lceil a / k\rceil+\lceil b / k\rceil$.

Recall that the bipartite Ramsey number for multicolorings $b_{k}(H)$ is the minimum $n$ such that every $k$-coloring of $E\left(K_{n, n}\right)$ yields a monochromatic copy of $H$. Analogous to the case with the classical Ramsey numbers, determining these numbers is hard. Chvátal [5], and Bieneke-Schwenk [3] proved that when $H=K_{p, q}$, this number is at most $(q-$ 1) $k^{p}+O\left(k^{p-1}\right)$, and some exact results for the case $H=K_{2, q}$ were also obtained in [3].

It is worth noting that the function $f_{2}^{k}\left(K_{a, b}\right)$ seems fundamentally different (and much easier to determine) from the numbers $b_{k}\left(K_{p, q}\right)$, since we do not require our complete bipartite subgraphs to have a specified number of vertices in each partite set. 


\section{Diameter at least four}

In this section we consider $f_{d}^{k}\left(K_{n}\right)$. Since a 1-subgraph is a clique, the problem is hopeless if $d=1$. The case $d=2$ was settled in [10], where nontrivial constructions were obtained that matched the trivial lower bounds asymptotically. We investigate the problem for larger $d$. We include the following slight strengthening of a well-known (and easy) fact for completeness (see problem 2.1.34 of [15]).

Proposition 10 Every 2-coloring of $E\left(K_{n}\right)$ yields a monochromatic spanning 2-subgraph or a monochromatic spanning 3-subgraph in each color. Thus in particular, $f_{d}^{2}\left(K_{n}\right)=n$ for $d \geq 3$.

Proof: Suppose that the coloring uses red and blue. We may assume that both the red subgraph and the blue subgraph have diameter at least three. Thus there exist vertices $r_{1}, r_{2}$ (respectively, $b_{1}, b_{2}$ ) with the shortest red $r_{1}, r_{2}$-path (respectively, blue $b_{1}, b_{2}$-path) having length at least three. We will show that the blue subgraph has diameter at most three.

Let $u, v$ be arbitrary vertices in $K_{n}$. If $\{u, v\} \cap\left\{r_{1}, r_{2}\right\} \neq \emptyset$, then the fact that there is no red $r_{1}, r_{2}$-path of length at most two guarantees a blue $u, v$-path of length at most two. We may therefore assume that $\{u, v\} \cap\left\{r_{1}, r_{2}\right\}=\emptyset$.

At least one of $u r_{1}, u r_{2}$ is blue, and at least one of $v r_{1}, v r_{2}$ is blue. Together with the blue edge $r_{1} r_{2}$, these three blue edges contain a $u, v$-path of length at most three. Since $u$ and $v$ are arbitrary, the blue subgraph has diameter at most three. Similarly, the vertices $b_{1}, b_{2}$ can be used to show that the red subgraph also has diameter at most three.

We now turn to the case when $d, k \geq 3$. The following $k$-coloring of $K_{n}$ has the property that the largest connected monochromatic subgraph has order $2\lceil n /(k+1)\rceil$ when $k$ is odd and $2\lceil n / k\rceil$ when $k$ is even. As we will see below, this is sharp when $k=3$, but not for any other value of $k$ when $k-1$ is a prime power [4].

This construction was suggested independently by Erdős. It uses the well-known fact that the edge-chromatic number of $K_{n}$ is $n$ if $n$ is odd and $n-1$ if $n$ is even.

Construction 11 When $k$ is odd, partition $V\left(K_{n}\right)$ into $k+1$ sets $V_{1}, \ldots, V_{k+1}$, each of size $\lfloor n /(k+1)\rfloor$ or $\lceil n /(k+1)\rceil$. Contract each $V_{i}$ to a single vertex $v_{i}$, and the edges between any pair $V_{i}, V_{j}$ to a single edge $v_{i} v_{j}$ to obtain $K_{k+1}$. Let $c: E\left(K_{k+1}\right) \rightarrow[k]$ be a proper edge-coloring. Expand $K_{k+1}$ back to the original $K_{n}$, coloring every edge between $V_{i}$ and $V_{j}$ with $c\left(v_{i} v_{j}\right)$. Color all edges within each $V_{i}$ with color 1 .

Because $c$ is a proper edge-coloring, a monochromatic connected graph $G$ can have $V(G) \cap V_{i} \neq \emptyset$ for at most two distinct indices $i \in[k]$. Thus $|V(G)| \leq 2\lceil n /(k+1)\rceil$. In the case $n \equiv 1(\bmod k)$, only one $V_{i}$ has size $\lceil n /(k+1)\rceil$ and all the rest have size $\lfloor n /(k+1)\rfloor$, so $|V(G)| \leq\lceil n /(k+1)\rceil+\lfloor n /(k+1)\rfloor$.

When $k$ is even, partition $V\left(K_{n}\right)$ into $k$ sets, color as described above with $k-1$ colors and change the color on any single edge to the $k$ th color. 
Proof of Theorem 6: For the upper bounds we use Construction 11. When $n \equiv 0,3$ $(\bmod 4), 2\lceil n / 4\rceil=\lceil n / 2\rceil$. When $n \equiv 2(\bmod 4), 2\lceil n / 4\rceil=n / 2+1$. When $n \equiv 1(\bmod$ $4)$, the construction gives the improvement $\lceil n / 4\rceil+\lfloor n / 4\rfloor$ which again equals the claimed bound $\lceil n / 2\rceil$.

For the lower bound, consider a 3-coloring $c: E\left(K_{n}\right) \rightarrow[3]$. Pick any vertex $v$, and assume without loss of generality that $\max \left\{d_{i}(v)\right\}=d_{1}(v)$. Let $N=v \cup N_{1}(v)$ and let $N^{\prime}=\left(\cup_{w \in N} N_{1}(w)\right)-N$. The subgraph in color 1 induced by $N \cup N^{\prime}$ is a 4-subgraph, thus we are done unless $|N|+\left|N^{\prime}\right| \leq n / 2$, which we may henceforth assume.

Let $M=V\left(K_{n}\right)-N-N^{\prime}$. Observe that color 1 is forbidden on edges between $N$ and $M$. Since $M \subseteq N_{2}(v) \cup N_{3}(v)$, we may assume without loss of generality that the set $S=N_{2}(v) \cap M$ satisfies $|S| \geq|M| / 2 \geq n / 4$.

If every $x \in N$ has the property that there is a $y \in S$ with $c(x y)=2$, then the subgraph in color 2 induced by $N \cup S$ is a 4 -subgraph with at least $(n+2) / 3+n / 4$ vertices, and we are done. We may therefore suppose that there is an $x \in N$ such that $c\left(x x^{\prime}\right)=3$ for every $x^{\prime} \in S$. For $i=2,3$, let

$$
A_{i}=\left\{u \in N \cup N^{\prime} \cup(M-S): \text { there is a } u^{\prime} \in S \text { with } c\left(u u^{\prime}\right)=i\right\} .
$$

By the definitions of $N, M$, and $A_{i}$, we have $A_{2} \cup A_{3} \supseteq N$. We next strengthen this to $A_{2} \cup A_{3} \supseteq N \cup N^{\prime}$. If there is a vertex $z \in N^{\prime}$ with $c(z y)=1$ for every $y \in S$, then the subgraph in color 1 induced by $S \cup N \cup\{z\}$ is a monochromatic 4-subgraph on at least $n / 4+(n+2) / 3+1 \geq n / 2+1$ vertices. Therefore we assume the $A_{2} \cup A_{3} \supseteq N \cup N^{\prime}$.

Because of $v$ and $x$, each of the sets $A_{i} \cup S$ induces a monochromatic 4-subgraph. Consequently, there is a monochromatic 4-subgraph of order at least $|S|+\max _{i}\left\{\left|A_{i}\right|\right\}$. By the previous observations, this is at least

$$
\begin{gathered}
|S|+\frac{\left|A_{2}\right|+\left|A_{3}\right|}{2} \geq\left\lceil\frac{|M|}{2}\right\rceil+\left\lceil\frac{\left|A_{2} \cup A_{3}\right|}{2}\right\rceil \geq \\
\geq\left\lceil\frac{|M|}{2}\right\rceil+\left\lceil\frac{\left|N \cup N^{\prime}\right|}{2}\right\rceil=\left\lceil\frac{|M|}{2}\right\rceil+\left\lceil\frac{n-|M|}{2}\right\rceil \geq\left\lceil\frac{n}{2}\right\rceil .
\end{gathered}
$$

We now improve this bound by one when $n=4 l+2$. We obtain the improvement unless equality holds above, which forces $|M|$ to be even, $|S|=|M| / 2$, and $A_{2} \cup A_{3}=N \cup N^{\prime}$. Recall that $|N|+\left|N^{\prime}\right| \leq n / 2$, which implies that $|M| \geq n / 2=2 l+1$. Because $|M|$ is even, we obtain $|M| \geq 2 l+2=n / 2+1$.

Since $A_{2} \cup A_{3}=N \cup N^{\prime}$, every vertex in $M-S$ has no edge to $S$ in color 2 or 3 . Thus all edges between $S$ and $M-S$ are of color 1, and the complete bipartite graph $B$ with parts $S$ and $M-S$ is monochromatic. Because $|S|=|M| / 2$, both $S$ and $M-S$ are nonempty. This implies that $B$ is a monochromatic 2 -subgraph with $|M| \geq n / 2+1$ vertices. 


\section{$5 \quad$ Diameter three and infinity}

In this section we prove Theorem 7 and also present an unpublished construction of Calkin which improves the bounds given by Construction 11 when $k-1>3$ is a prime power.

Proof of Theorem 7: Given a $k$-coloring $c: E\left(K_{n}\right) \rightarrow[k]$, choose $v \in V\left(K_{n}\right)$, and assume that $d_{i}(v)$ is maximized when $i=1$. Consider the bipartite graph $G$ with bipartition $A=v \cup N_{1}(v)$ and $B=V\left(K_{n}\right)-A$; set $a=|A|$. For $x \in A$ and $y \in B$, let $x y \in E(G)$ if $c(x y) \neq 1$. Let $\Delta=\max _{w \in A}\left|N_{1}(w) \cap B\right|$. Then $E(G) \geq a(n-a-\Delta)$.

For any $w \in A$ with $\left|N_{1}(w) \cap B\right|=\Delta$, the subgraph in color 1 induced by $A \cup N_{1}(w)$ is a 3 -subgraph with at least $a+\Delta$ vertices. By definition, color 1 is absent in $G$ and thus $E(G)$ is $(k-1)$-colored. Lemma 8 applied to $G$ yields a 3 -subgraph on at least $(n-a-\Delta) /(k-1)+a(n-a-\Delta) /((k-1)(n-a))$ vertices. Thus $K_{n}$ contains a 3 -subgraph of order at least

$$
\min _{\substack{a, \Delta \\ a \geq 1+(n-1) / k \\ \Delta \leq n-a}} \max \left\{a+\Delta, \frac{a(n-a-\Delta)}{k-1}\left(\frac{1}{a}+\frac{1}{n-a}\right)\right\} .
$$

We let $\Delta$ and $a$ take on real values to obtain a lower bound on this minimum. Since one of these functions is increasing in $\Delta$ and the other is decreasing in $\Delta$, the choice of $\Delta$ that minimizes the maximum (for fixed $a$ ) is that for which the two quantities are equal. This choice is

$$
\Delta=\frac{(n-a)(n-a(k-1))}{k n-a(k-1)},
$$

and both functions become $n^{2} /(k n-a(k-1))$. Since this is an increasing function for $1+(n-1) / k \leq a<k n /(k-1)$, and since we are assuming $a \leq n$, the minimum is obtained at $a=1+(n-1) / k$. This yields a lower bound of $k n^{2} /\left(\left(k^{2}-k+1\right) n-(k-1)^{2}\right)$.

Definition 12 For a positive integer $k$, let $f_{\infty}^{k}(G)$ be the maximum $t$ with the property that every $k$-coloring of $E(G)$ yields a monochromatic connected subgraph on at least $t$ vertices.

Clearly $f_{d}^{k}(G) \leq f_{\infty}^{k}(G)$ for each $d$, since a $d$-subgraph is connected. Construction 11 and Theorem 6 therefore immediately yield $f_{\infty}^{3}\left(K_{n}\right)=n / 2+1$ or $\lceil n / 2\rceil$ depending on whether $n \equiv 2(\bmod 4)$ or not (see also exercise 14 of Chapter 6 of [1]). For larger $k$, however, the following unpublished construction due to Calkin improves Construction 11

Construction 13 (Calkin) Let $q$ be a prime power and $\mathbf{F}$ be a finite field on $q$ elements. We exhibit a $q+1$-coloring of $E\left(K_{q^{2}}\right)$. Let $V\left(K_{q^{2}}\right)=\mathbf{F} \times \mathbf{F}$. Color the edge $(i, j)\left(i^{\prime}, j^{\prime}\right)$ by the field element $\left(j^{\prime}-j\right) /\left(i^{\prime}-i\right)$ if $i \neq i^{\prime}$, and color all edges $(i, j)\left(i, j^{\prime}\right)$ with a single new color. This coloring is well-defined since $\left(j^{\prime}-j\right) /\left(i^{\prime}-i\right)=\left(j-j^{\prime}\right) /\left(i-i^{\prime}\right)$. 
Lemma 14 Construction 13 produces a $q+1$-coloring of $E\left(K_{q^{2}}\right)$ such that the subgraph of any given color consists of $q$ vertex disjoint copies of $K_{q}$.

Proof: This is certainly true of the color on edges of the form $(i, j)\left(i, j^{\prime}\right)$. Now fix a color $l \in \mathbf{F}$. Let $(x, y) \sim\left(x^{\prime}, y^{\prime}\right)$ if the edge $(x, y)\left(x^{\prime}, y^{\prime}\right)$ has color $l$. We will show that this relation is transitive.

Suppose that $(i, j) \sim\left(i^{\prime}, j^{\prime}\right)$ and $\left(i^{\prime}, j^{\prime}\right) \sim\left(i^{\prime \prime}, j^{\prime \prime}\right)$. Then

$$
\left(j^{\prime}-j\right) /\left(i^{\prime}-i\right)=l=\left(j^{\prime \prime}-j^{\prime}\right) /\left(i^{\prime \prime}-i^{\prime}\right) .
$$

Consequently,

$$
\left(j^{\prime \prime}-j\right)=\left(j^{\prime \prime}-j^{\prime}\right)+\left(j^{\prime}-j\right)=l\left(i^{\prime \prime}-i^{\prime}\right)+l\left(i^{\prime}-i\right)=l\left(i^{\prime \prime}-i\right)
$$

and therefore $(i, j) \sim\left(i^{\prime \prime}, j^{\prime \prime}\right)$.

Since this relation on $V\left(K_{q^{2}}\right) \times V\left(K_{q^{2}}\right)$ is an equivalence relation, the edges in color $l$ form vertex disjoint complete graphs. For fixed $i, j, l$, there are exactly $q-1$ distinct $(x, y) \neq(i, j)$ for which $(x, y) \sim(i, j)$, because $x \neq i$ uniquely determines $y$. This completes the proof.

Lemma 14 together with Theorem 5 allows us to easily obtain good bounds for $f_{\infty}^{k}\left(K_{n}\right)$. The author believes that the following theorem was also proved independently by Calkin. Our proof of the lower bound given below uses Theorem 5 .

Theorem 15 Let $k-1$ be a prime power. Then $n /(k-1) \leq f_{\infty}^{k}\left(K_{n}\right) \leq n /(k-1)+k-1$.

Proof: For the upper bound we use the idea of Construction 13. Let $\mathbf{F}$ be a finite field of $q=k-1$ elements. Partition $V\left(K_{n}\right)$ into $(k-1)^{2}$ sets $V_{i, j}$ of size $\left\lfloor n /(k-1)^{2}\right\rfloor$ or $\left\lceil n /(k-1)^{2}\right\rceil$, where $i, j \in \mathbf{F}$. Color all edges between $V_{i, j}$ and $V_{i^{\prime}, j^{\prime}}$ by the field element $\left(j^{\prime}-j\right) /\left(i^{\prime}-i\right)$ if $i \neq i^{\prime}$, and by a new color if $i=i^{\prime}$. Color all edges within each $V_{i, j}$ by a single color in $\mathbf{F}$.

Lemma 14 implies that the order of the largest monochromatic connected subgraph is at most $\left\lceil n /(k-1)^{2}\right\rceil(k-1) \leq n /(k-1)+k-1$.

For the lower bound, consider a $k$-coloring of $E\left(K_{n}\right)$. We may assume that the subgraph $H$ in some color $l$ is not a connected spanning subgraph. This yields a partition $X \cup Y$ of $V\left(K_{n}\right)$ such that no edge between $X$ and $Y$ has color $l$ (let $X$ be a component of $H)$. The bipartite graph $B$ formed by the $X, Y$ edges is colored with $k-1$ colors. Applying Theorem 5 to $B$ yields a 3 -subgraph of order at least $|X| /(k-1)+|Y| /(k-1)=n /(k-1)$. 


\section{Table of Results}

Table of Results for $f_{d}^{k}\left(K_{n}\right)$

\begin{tabular}{|c|c|c|c|c|c|}
\hline$d$ & 2 & 3 & 4 & 5 & \\
\hline 1 & \multicolumn{5}{|c|}{ Equivalent to classical Ramsey numbers } \\
\hline 2 & $\left\lceil\frac{3 n}{4}\right\rceil, \quad\lceil 10]$ & \multicolumn{4}{|c|}{$\sim \frac{n}{k}, \quad[10]$} \\
\hline 3 & $\begin{array}{c}n, \\
\text { Proposition } 10\end{array}$ & $\begin{array}{l}\quad \leq\left\lceil\frac{n}{2}\right\rceil+1, \\
\text { Construction } 11 \\
>\frac{3 n}{7}, \text { Theorem } 7\end{array}$ & $\begin{array}{l}\quad \leq \frac{n}{3}+2, \\
\text { Construction } 13 \\
>\frac{4 n}{13}, \text { Theorem } 7\end{array}$ & $\begin{aligned} \leq & \frac{n}{k-1}+k-1 \\
& \text { power, Cons } \\
> & \frac{n}{k-1+1 / k}\end{aligned}$ & $\begin{array}{l}k-1 \text { prime } \\
\text { Thuction } 13 \\
\text { Theorem } 7\end{array}$ \\
\hline$\vdots$ & & $\begin{array}{c}\left\lceil\frac{n}{2}\right\rceil \text { or }\left\lceil\frac{n}{2}\right\rceil+1 \\
\text { Construction } 11 \\
\text { Theorem } 6\end{array}$ & $\begin{array}{l}\qquad \leq \frac{n}{3}+3 \\
\text { Construction } 13\end{array}$ & $\begin{array}{l}\quad \leq \frac{n}{4}+4 \\
\text { Construction } 13\end{array}$ & \\
\hline
\end{tabular}

\section{Acknowledgments}

The author thanks Tom Fowler for informing him about [10], and an anonymous referee for informing him about [4]. Thanks also to Annette Rohrs for help with typesetting the article.

\section{References}

[1] B. Bollobás, Modern Graph Theory, Springer-Verlag (1998).

[2] S. Burr, P. Erdős, Generalizations of a Ramsey-theoretic result of Chvátal, J. Graph Theory 7 (1983), no. 1, 39-51.

[3] L. W. Beineke, A. J. Schwenk, On a bipartite form of the Ramsey problem, Proceedings of the Fifth British Combinatorial Conference (Univ. Aberdeen, Aberdeen, 1975), pp. 17-22. Congressus Numerantium, No. XV, Utilitas Math., Winnipeg, Man., 1976.

[4] N. Calkin, unpublished. 
[5] V. Chvátal, On finite polarized partition relations, Canad. Math. Bul., 12, (1969), 321-326.

[6] M. K. Chung, C. L. Liu, A generalization of Ramsey theory for graphs, Discrete Math. 21 (1978), no. 2, 117-127.

[7] G. Chen, R. H. Schelp, Ramsey problems with bounded degree spread, Combin. Probab. Comput. 2 (1993), no. 3, 263-269.

[8] P. Erdős, personal communication with T. Fowler.

[9] P. Erdős, A. Hajnal, J. Pach, On a metric generalization of Ramsey's theorem, Israel J. Math. 102 (1997), 283-295.

[10] T. Fowler, Finding large monochromatic diameter two subgraphs, to appear.

[11] A. Gyárfás, Fruit salad, Electron. J. Combin. 4 (1997), no. 1, Research Paper 8, 8 pp. (electronic).

[12] M. S. Jacobson, On a generalization of Ramsey theory, Discrete Math. 38 (1982), no. 2-3, 191-195.

[13] J. Nešetruil, V. Rödl A structural generalization of the Ramsey theorem, Bull. Amer. Math. Soc. 83 (1977), no. 1, 127-128.

[14] R. N. Tonoyan, An analogue of Ramsey's theorem, Applied mathematics, No. 1 (Russian), 61-66, 92-93, Erevan. Univ., Erevan, 1981.

[15] D. B. West, Introduction to Graph Theory, Prentice Hall, Inc., (1996). 